\title{
Josephson flux-flow oscillators in nonuniform microwave fields
}

\section{Salerno, Mario; Samuelsen, Mogens Rugholm}

\section{Published in:}

Physical Review B Condensed Matter

Link to article, DOI:

10.1103/PhysRevB.61.99

Publication date:

2000

Document Version

Publisher's PDF, also known as Version of record

Link back to DTU Orbit

Citation (APA):

Salerno, M., \& Samuelsen, M. R. (2000). Josephson flux-flow oscillators in nonuniform microwave fields. Physical Review B Condensed Matter, 61(1), 99-102. https://doi.org/10.1103/PhysRevB.61.99

\section{General rights}

Copyright and moral rights for the publications made accessible in the public portal are retained by the authors and/or other copyright owners and it is a condition of accessing publications that users recognise and abide by the legal requirements associated with these rights.

- Users may download and print one copy of any publication from the public portal for the purpose of private study or research.

- You may not further distribute the material or use it for any profit-making activity or commercial gain

- You may freely distribute the URL identifying the publication in the public portal

If you believe that this document breaches copyright please contact us providing details, and we will remove access to the work immediately and investigate your claim. 


\title{
Josephson flux-flow oscillators in nonuniform microwave fields
}

\author{
Mario Salerno* \\ Dipartimento di Scienze Fisiche “E.R. Caianiello,' Universitá di Salerno, I-84100 Salerno, Italy \\ M. R. Samuelsen \\ Department of Physics, The Technical University of Denmark, DK-2800 Lyngby, Denmark
}

(Received 2 April 1999; revised manuscript received 21 September 1999)

\begin{abstract}
We present a simple theory for Josephson flux-flow oscillators in the presence of nonuniform microwave fields. In particular we derive an analytical expression for the $I-V$ characteristic of the oscillator from which we show that satellite steps are spaced around the main flux-flow resonance by only even harmonics of the rf frequency. This result is found to be in good agreement with our numerical results and with experiments.
\end{abstract}

In the past years a great deal of interest has been devoted to the study of Josephson flux-flow oscillators, i.e., long Josephson junctions operating in the flux-flow regime. ${ }^{1-10}$ This is mainly due to their high output power, wide bandwidth, and easy tunability, these being attractive features for applications in superconducting millimeter-wave electronics. ${ }^{11,12}$ The dynamical states characterizing the flux-flow regime produce in the current-voltage $(I-V)$ characteristic a highvoltage step (flux-flow step) split into equally spaced Fiske substeps. The application of an external rf field to the junction gives rise to interesting phenomena such as the appearance of satellite steps around the main flux-flow resonance. It was recently shown ${ }^{10}$ that $\mathrm{rf}$ fields applied uniformly along the junction give rise in the $I-V$ characteristic to both even and odd satellite steps. On the other hand it is known, both from numerical and experimental studies, ${ }^{7,8}$ that for rf fields applied at the edges of the junction, only even satellite steps are present. This raises the question: why for nonuniform microwave fields are the odd satellite steps missing?

The aim of the present paper is to answer this question by providing a theory for the satellite steps of a Josephson fluxflow oscillator in the presence of a nonuniform rf field applied through boundary conditions. To this end we use a perturbative expansion around the uniform rotating solution to derive an analytical expression for the $I-V$ curve. As a result we show that satellite steps appear at positions shifted from the main resonance only by even multiples of the applied rf frequency. The heights of these resonances are modulated by the rf field and can be changed by increasing the amplitude of the microwave field. To check these results we have compared our analytical expression for the $I-V$ characteristic with the results of numerical integrations, obtaining an excellent agreement between theory and numerical experiment.

The paper is organized as follows. In Sec. I we introduce the model for the flux-flow oscillator in the presence of nonuniform microwave fields, and use a perturbative expansion around the uniform rotating background solution to derive an analytical expression for the $I-V$ curve. In Sec. II we compare analytical results with direct numerical simulations of the perturbed sine-Gordon system and summarize the main results of the paper.

\section{FLUX-FLOW OSCILLATORS IN NONUNIFORM MICROWAVE FIELDS}

The electrodynamics of a Josephson flux-flow oscillator in the presence of both microwaves and external magnetic fields at the edges of the junction is described by the perturbed sine-Gordon equation, ${ }^{11,12}$

$$
\Phi_{x x}-\Phi_{t t}=\sin (\Phi)+\alpha \Phi_{t}-\eta,
$$

with the boundary conditions

$$
\begin{aligned}
& \Phi_{x}(0, t)=\Gamma+\Gamma_{1} \sin (\Omega t), \\
& \Phi_{x}(L, t)=\Gamma+\Gamma_{2} \sin (\Omega t) .
\end{aligned}
$$

In Eq. (1) space and time have been normalized to the Josephson penetration length $\lambda_{J}$ and to the inverse plasma frequency $\omega_{0}^{-1}$, respectively. $\alpha$ denotes the loss parameter associated to the quasiparticle tunneling, $\eta$ represents the dc bias current, and $\Gamma$ is the normalized external magnetic field. $\Gamma_{1}, \Gamma_{2}$ are the normalized rf magnetic field at the two ends of the junction and $\Omega$ its normalized frequency. To describe the dynamics of the junction in the flux-flow regime we assume a solution for the field equation (1) of the form

$$
\Phi=\omega t+\Gamma x+f(x) \sin (\Omega t)+g(x) \cos (\Omega t)+\Psi(x, t)+\theta_{1},
$$

where $\Psi$ is a small field ( $\Psi \ll 1), \theta_{1}$ is an arbitrary phase, and $f(x), g(x)$ are unknown functions that satisfy the boundary conditions

$$
g^{\prime}(0)=g^{\prime}(L)=0, f^{\prime}(0)=\Gamma_{1}, f^{\prime}(L)=\Gamma_{2}
$$

(here a prime denotes $x$ derivative). Note that these conditions are consistent with the ones in Eq. (2) if

$$
\Psi_{x}(0, t)=\Psi_{x}(L, t)=0
$$

In the following we look for fields $\Psi$, which satisfy Eq. (5) and have both zero space and time averages $\langle\Psi\rangle=\Psi=0$ (we denote with \langle\rangle and with the overbar, respectively, time and space averages). Inserting Eq. (3) into Eq. (1) and using the smallness of $\Psi$ we get the linearized equation 


$$
\begin{aligned}
\Psi_{x x}-\Psi_{t t}-\alpha \Psi_{t}= & \alpha \omega-\eta-\left[f^{\prime \prime}(x)+f(x) \Omega^{2}\right. \\
& +\alpha g(x) \Omega] \sin \Omega t-\left[g^{\prime \prime}(x)+g(x) \Omega^{2}\right. \\
& -\alpha f(x) \Omega] \cos \Omega t+\sin [\Gamma x+\omega t \\
& \left.+f(x) \sin \Omega t+g(x) \cos \Omega t+\theta_{1}\right] \\
& +\cos [\Gamma x+\omega t+f(x) \sin \Omega t \\
& \left.+g(x) \cos \Omega t+\theta_{1}\right] \Psi
\end{aligned}
$$

It is convenient to eliminate the explicit $\Omega$ dependence from this equation by taking $f(x)$ and $g(x)$ to be solutions of the system

$$
\begin{aligned}
& f^{\prime \prime}(x)+\Omega^{2} f(x)+\alpha \Omega g(x)=0, \\
& g^{\prime \prime}(x)+\Omega^{2} g(x)-\alpha \Omega f(x)=0,
\end{aligned}
$$

satisfying the boundary conditions in Eq. (4). One can check that this boundary value problem can be solved as

$$
\begin{aligned}
& f(x)=f_{a}\left(z+\frac{L}{2}\right)-f_{s}\left(z+\frac{L}{2}\right), \\
& g(x)=g_{a}\left(z+\frac{L}{2}\right)-g_{s}\left(z+\frac{L}{2}\right),
\end{aligned}
$$

where

$$
\begin{aligned}
& f_{s}(z)=-\lambda \mathbf{s}^{t r}\left(\frac{L}{2}\right) \cdot \mathbf{M}^{-1} \cdot \mathbf{a}(z), \\
& g_{s}(z)=\lambda \mathbf{s}^{t r}\left(\frac{L}{2}\right) \cdot \mathbf{i} \cdot \mathbf{M}^{-1} \cdot \mathbf{a}(z),
\end{aligned}
$$

with $z=x-L / 2$ and $\mathbf{s}(z), \mathbf{a}(z)$, denoting, respectively, symmetric and asymmetric (in the coordinate $z$ ) vector solutions of the fourth-order differential equation $\left[\left(\partial_{x}^{2}+\Omega^{2}\right)^{2}\right.$ $\left.+\alpha^{2} \Omega^{2}\right] F(x)=0$ :

$$
\begin{aligned}
& \mathbf{s}^{t r}(z)=\left(\cos \Omega_{1} z \cosh \frac{\alpha_{1} z}{2}, \sin \Omega_{1} z \sinh \frac{\alpha_{1} z}{2}\right), \\
& \mathbf{a}^{t r}(z)=\left(\cos \Omega_{1} z \sinh \frac{\alpha_{1} z}{2}, \sin \Omega_{1} z \cosh \frac{\alpha_{1} z}{2}\right)
\end{aligned}
$$

(here $t r$ means transpose). In Eq. (9) $\lambda \mathbf{s}$ is given by

$$
\lambda=-\frac{\Gamma_{0}}{\mathbf{s}^{t r}\left(\frac{L}{2}\right) \cdot \mathbf{s}\left(\frac{L}{2}\right)} .
$$

i is the $2 \times 2$ asymmetric unit matrix, $M^{-1}$ is the inverse of the matrix $M$ with elements $m_{11}=m_{22}=\alpha_{1} / 2, m_{21}=-m_{12}$ $=\Omega_{1}$, and $\alpha_{1}, \Omega_{1}$ are

$$
\begin{gathered}
\alpha_{1}=\left[2\left(\sqrt{\Omega^{4}+\alpha^{2} \Omega^{2}}-\Omega^{2}\right)\right]^{1 / 2}, \\
\Omega_{1}=\sqrt{\frac{\sqrt{\Omega^{4}+\alpha^{2} \Omega^{2}}+\Omega^{2}}{2}} .
\end{gathered}
$$

The expressions for $f_{a}(z), g_{a}(z)$ in Eq. (8) follow from those of $f_{s}(z), g_{s}(z)$ by simply interchanging a with $\mathbf{s}$ in Eqs. (9) and (11).

Knowing $f(x)$ and $g(x)$, and using the Bessel identity $e^{i \beta \sin (\Omega t)}=\sum_{m=-\infty}^{\infty} J_{m}(\beta) e^{i m \Omega t}$, we can rewrite Eq. (6) in the form

$$
\begin{aligned}
\Psi_{x x}-\Psi_{t t}-\alpha \Psi_{t}= & \alpha \omega-\eta+\sum_{m} J_{m}(h)\{\sin [\Gamma x+m \phi(x)] \\
& \times \cos \left(\tilde{\omega}_{m} t+\theta_{1}\right)+\cos [\Gamma x+m \phi(x)] \\
& \left.\times \sin \left(\tilde{\omega}_{m} t+\theta_{1}\right)\right\}+\sum_{m} J_{m}(h)\{\cos [\Gamma x \\
& +m \phi(x)] \cos \left(\tilde{\omega}_{m} t+\theta_{1}\right)-\sin [\Gamma x \\
& \left.+m \phi(x)] \sin \left(\tilde{\omega}_{m} t+\theta_{1}\right)\right\} \Psi
\end{aligned}
$$

where $\tilde{\omega}_{m}=\omega+m \Omega$, and $h, \phi(x)$ are given by

$$
h=\sqrt{f(x)^{2}+g(x)^{2}}, \quad \tan \phi(x)=\frac{g(x)}{f(x)} .
$$

At a given frequency $\omega \neq-m \Omega$, the third term on the righthand side (rhs) of Eq. (13) will excite the frequencies $\widetilde{\omega}_{m}$ in $\Psi$. This will generate, via the last term on the rhs of Eq. (13), second-order terms at frequencies $\tilde{\omega}_{m_{1}} \pm \widetilde{\omega}_{m_{2}}$, i.e., at $2 \omega$ $+M \Omega$ and $M \Omega$, which in the following will be neglected. A special situation occurs when $\tilde{\omega}_{m}=0$. Then all frequencies will be multiples of $\Omega$ and phase locking will appear. ${ }^{13}$ To solve Eq. (13) we assume $\omega$ and $\Omega$ to be incommensurable (i.e., no phase locking) and expand the function $\Psi$ as

$$
\Psi=\sum_{n, m}\left[C_{n m} \cos \left(\tilde{\omega}_{m} t+\theta_{1}\right)+D_{n m} \sin \left(\tilde{\omega}_{m} t+\theta_{1}\right)\right] \cos k_{n} x
$$

with $k_{n}=(\pi / L) n$ [note that with this choice the boundary conditions in Eq. (5) are automatically satisfied]. By substituting the above expansion into Eq. (13) and projecting along the $k_{n}, \tilde{\omega}_{m}$, modes we get

$$
C_{n m}=\frac{\left(\tilde{\omega}_{m}^{2}-k_{n}^{2}\right) \tilde{S}_{n m}+\alpha \tilde{\omega}_{m} \widetilde{C}_{n m}}{\left[\left(\tilde{\omega}_{m}^{2}-k_{n}^{2}\right)^{2}+\alpha^{2} \tilde{\omega}_{m}^{2}\right]}
$$

$$
D_{n m}=\frac{\left(\tilde{\omega}_{m}^{2}-k_{n}^{2}\right) \tilde{C}_{n m}-\alpha \tilde{\omega}_{m} \tilde{S}_{n m}}{\left[\left(\tilde{\omega}_{m}^{2}-k_{n}^{2}\right)^{2}+\alpha^{2} \tilde{\omega}_{m}^{2}\right]}
$$

where

$$
\begin{aligned}
& \tilde{C}_{n m}=\frac{1}{L} \int_{0}^{L} \cos \left(k_{n} x\right) J_{m}(h) \cos [\Gamma x+m \phi(x)] d x, \\
& \tilde{S}_{n m}=\frac{1}{L} \int_{0}^{L} \cos \left(k_{n} x\right) J_{m}(h) \sin [\Gamma x+m \phi(x)] d x .
\end{aligned}
$$

The $I-V$ characteristic then follows from the dc part of Eq. (13) (where now the last term of the rhs contributes) as 


$$
\begin{aligned}
\eta= & \alpha \omega+\frac{1}{2} \sum_{n, m}\left(C_{n m} \widetilde{C}_{n m}-D_{n m} \tilde{S}_{n m}\right) \\
= & \alpha \omega+\frac{1}{2} \sum_{n, m}\left[\widetilde{C}_{n m}^{2}+\tilde{S}_{n m}(n, m)^{2}\right] \\
& \times \frac{\alpha \tilde{\omega}_{m}}{\left(\tilde{\omega}_{m}^{2}-k_{n}^{2}\right)^{2}+\alpha^{2} \tilde{\omega}_{m}^{2}}
\end{aligned}
$$

[note that in deriving Eq. (19) we used $\tilde{\omega}_{m} \neq 0$ ]. This expression, although exact, is quite complicated to analyze without resorting to numerical tools. One can simplify it by making the assumption $\pi / L \ll \Omega \ll \omega, \Gamma$, so that the integrals $\widetilde{C}_{n m}$ and $\tilde{S}_{n m}$ in Eq. (19) can be approximated as

$$
\begin{aligned}
& \widetilde{C}_{n m} \simeq C(n, \Gamma) B(m, C)-S(n, \Gamma) B(m, S), \\
& \widetilde{S}_{n m} \simeq S(n, \Gamma) B(m, C)+C(n, \Gamma) B(m, S),
\end{aligned}
$$

with

$$
\begin{aligned}
& B(m, C)=\frac{1}{L} \int_{0}^{L} J_{m}(h) \cos [m \phi(x)] d x, \\
& B(m, S)=\frac{1}{L} \int_{0}^{L} J_{m}(h) \sin [m \phi(x)] d x,
\end{aligned}
$$

and

$$
\begin{aligned}
C(n, \Gamma) & =\frac{1}{L} \int_{0}^{L} \cos \left(k_{n} x\right) \cos (\Gamma x) d x \\
& =\frac{\cos \left(k_{n} L\right) \Gamma L \sin (\Gamma L)}{(\Gamma L)^{2}-\left(k_{n} L\right)^{2}}, \\
S(n, \Gamma) & =\frac{1}{L} \int_{0}^{L} \cos \left(k_{n} x\right) \sin (\Gamma x) d x \\
& =\frac{\Gamma L\left[1-\cos \left(k_{n} L\right) \cos (\Gamma L)\right]}{(\Gamma L)^{2}-\left(k_{n} L\right)^{2}} .
\end{aligned}
$$

Equation (19) is then rewritten as

$$
\begin{aligned}
\eta= & \alpha \omega+\frac{1}{2} \sum_{n, m}\left[C(n, \Gamma)^{2}+S(n, \Gamma)^{2}\right]\left[B(m, C)^{2}\right. \\
& \left.+B(m, S)^{2}\right] \frac{\alpha \tilde{\omega}_{m}}{\left[\left(\tilde{\omega}_{m}^{2}-k_{n}^{2}\right)^{2}+\alpha^{2} \tilde{\omega}_{m}^{2}\right]} .
\end{aligned}
$$

A further simplification is achieved by observing that the integrals in Eq. (22) can be put in the form ${ }^{14}$

$$
\begin{gathered}
B(m, C)=\frac{1}{L} \int_{0}^{L} \sum_{M}(-1)^{M} J_{m+2 M}(f) J_{2 M}(g) d x, \\
B(m, S)=\frac{1}{L} \int_{0}^{L} \sum_{M}(-1)^{M} J_{m+2 M+1}(f) J_{2 M+1}(g) d x .
\end{gathered}
$$

From the explicit solutions for $f(x)$ and $g(x)$ we see that these functions are almost harmonic and, for $L$ large, they oscillate many times in the interval $[0, L]$. This implies that the integrals $B(m, C), B(m, S)$, for odd values of $m$ are very small [the spatial variations of $f(x), g(x)$ average them out], i.e., $B(2 m+1, C) \simeq 0, B(2 m+1, S) \simeq 0$. Using these relations and the symmetry properties $B(-2 m, C)$ $=B(2 m, C), B(-2 m, S)=-B(2 m, S), B(0, S)=0$, we can finally approximate the $I-V$ curve as

$$
\begin{aligned}
\eta \simeq \alpha \omega+\sum_{n, m=-\infty}^{\infty} \frac{1}{2} X(2 m) \frac{\left(\frac{\Gamma L}{2}\right)^{2}}{\left(\frac{\Gamma L+k_{n} L}{2}\right)^{2}} \\
\quad \times \frac{\sin ^{2} \frac{\Gamma L-k_{n} L}{2}}{\left(\frac{\Gamma L-k_{n} L}{2}\right)^{2}} \frac{\alpha(\omega+2 m \Omega)}{\left[(\omega+2 m \Omega)^{2}-k_{n}^{2}\right]^{2}+\alpha^{2}(\omega+2 m \Omega)^{2}},
\end{aligned}
$$

where $X(2 m)=B(2 m, C)^{2}+B(2 m, S)^{2}$. Note that the sum on $n$ in this equation gives rise to Fiske resonances spaced by $\pi / L$ with the Fraunhofer factor enhancing the ones for which $k_{n}$ is close to $\Gamma+M \Omega, M=0, \pm 2, \pm 4, \ldots$. It is interesting that this approximate expression for the $I-V$ curve is similar to the exact (up to first order) expression derived in Ref. 10 for the case of uniform microwave fields, except for the fact that the sum on $m$ here is only on the even relative integers $M=2 m$. This implies that in the $I-V$ curve, only satellite steps at voltages $\omega=\Gamma+M \Omega$ can appear. We finally remark that the absence of the odd steps in the case of nonuniform fields is due to the spatial variation of the $f(x)$ and $g(x)$ functions inside the junction.

\section{NUMERICAL EXPERIMENT}

In order to check the analytical expressions for the $I-V$ characteristics derived in Eq. (19), we have numerically integrated Eq. (1) with the boundary conditions in Eq. (2). For fixed values of magnetic field, damping constant, amplitude, and frequency of the rf field and length of the junction, we computed the normalized average voltage across the junction $V=\overline{\left\langle\Phi_{t}\right\rangle} \equiv \omega$ as a function of the bias current, taking as initial condition $n=\Gamma L / 2 \pi$ fluxons equally spaced along the junction. The numerical $I-V$ characteristic was obtained by integrating Eq. (1) long enough to eliminate all transients and measuring for each value of the bias current the corresponding average voltage. To trace the $I-V$ curves the bias current was increased in small steps from $\eta=0$ to $\eta=1$ and then back to zero. At each $\eta$ step the final configuration of the field in the junction was used as initial condition for the next $\eta$ step.

In Fig. 1 we show the $I-V$ characteristic of a long Josephson junction in the presence of a symmetric rf field of amplitude $\Gamma_{1}=\Gamma_{2}=2.0$, and frequency $\Omega=1.8$, for parameter values $\alpha=0.1, L=10, \Gamma=6$. The thin line refers to the numerical evaluation of Eq. (19) while the thick one represents the numerical integration of Eq. (1). In this figure we see a flux-flow step at $\omega=\Gamma$ and two satellite steps at $\omega=\Gamma$ 


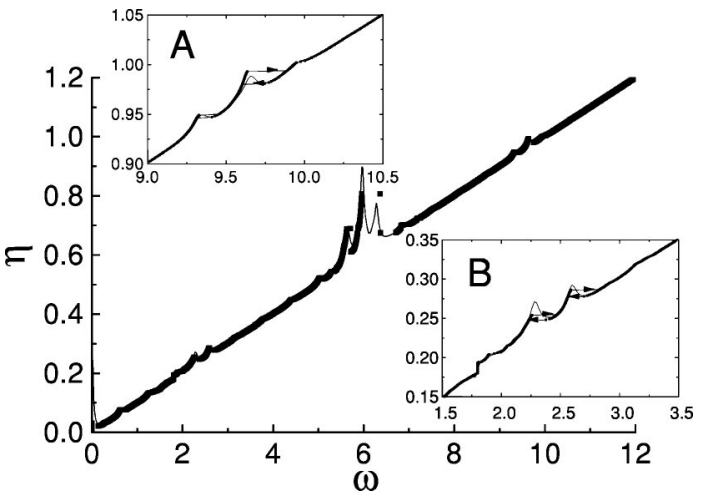

FIG. 1. The $I-V$ characteristic of a long Josephson junction in the presence of a nonuniform symmetric microwave field at the boundaries of amplitude $\Gamma_{1}=\Gamma_{2}=2.0$, frequency $\Omega=1.8$. The parameter values of the junction are $\alpha=0.1, L=10, \Gamma=6$. Insets $A, B$ show an enlargement of the satellite steps at $\omega=\Gamma+2 \Omega$ and $\omega$ $=\Gamma-2 \Omega$, respectively. The thin curve refers to the analytical expression in Eq. (19), while the thick one is obtained from numerical integrations of Eq. (1).

$\pm 2 \Omega$ but no satellite steps at $\omega=\Gamma \pm \Omega$. Moreover, the resonances are split in Fiske substeps spaced by $\pi / L$ in agreement with our analysis. Insets $A, B$ show an enlargement of the satellite steps at $\omega=\Gamma+2 \Omega$ and $\omega=\Gamma-2 \Omega$, respectively. In Fig. 2 we report the $I-V$ curve for the same parameters values as in Fig. 1 but for the amplitude of rf field at the boundaries $\Gamma_{1}=\Gamma_{2}=4.0$. We see that, with respect to Fig. 1, the size of the satellite steps has increased, while the one of the main resonance has decreased. Similar results are obtained also for the case of asymmetric of fields $\Gamma_{1}=-\Gamma_{2}$ applied at the boundaries. A general feature emerging from these calculations is the appearance of vertical phase-locked steps at voltages $\omega=M \Omega$ (and subharmonics), which coex-

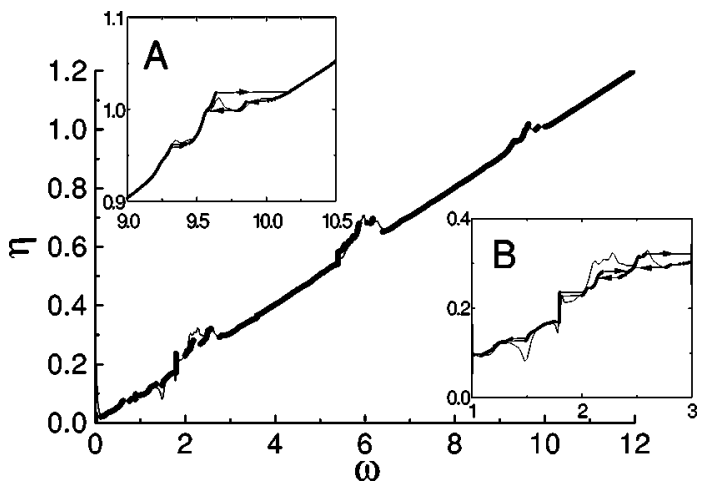

FIG. 2. Same as in Fig. 1 but for $\mathrm{rf}$ amplitude $\Gamma_{1}=\Gamma_{2}=4.0$.

ists with the flux flow satellite structures. By increasing the amplitude of the rf field, phase lock becomes more and more dominant with respect to flux flow. At higher fields the mixing of the phase lock with the flux flow will strongly influence the shape of satellites steps and the agreement between the theory and the numerical experiments usually becomes less accurate (see inset $B$ of Fig. 2). This is due to the interference of the two last terms in the rhs of Eq. (13), which was completely neglected in our analysis.

In conclusion, we have presented a theory for long Josephson flux-flow oscillators in the presence of microwave fields applied at the edges of the junction, which accounts for the appearance of satellite steps around the main flux-flow resonance. We derived an analytical expression for the $I-V$ characteristics and showed that only satellite steps spaced by even harmonics are present in the $I-V$ characteristic. We remark that this behavior is different from what we found in the case of uniform rf fields, and in turn can be useful to distinguish the type of microwave coupling realized in a real experiment.
*Also at Istituto Nazionale di Fisica della Materia (INFM) Unità di Salerno.

${ }^{1}$ T. Nagatsuma, K. Enpuku, F. Irie, and K. Yoshida, J. Appl. Phys. 54, 3302 (1983); 56, 3284 (1984).

${ }^{2}$ Y.M. Zhang and P.H. Wu, J. Appl. Phys. 68, 4703 (1990).

${ }^{3}$ A.V. Ustinov, J. Mygind, and V.A. Oboznov, J. Appl. Phys. 72, 1203 (1992).

${ }^{4}$ V.P. Koshelets, A.V. Shchukin, S.V. Shitov, and L.V. Filippenko, IEEE Trans. Appl. Supercond. 3, 2524 (1993).

${ }^{5}$ Y.M. Zhang, D. Winkler, and T. Claeson, Appl. Phys. Lett. 62, 3195 (1993).

${ }^{6}$ M. Cirillo, F. Santucci, P. Carelli, M.G. Castellano, and R. Leoni, J. Appl. Phys. 73, 8637 (1993).

${ }^{7}$ A.V. Ustinov, J. Mygind, N.F. Pedersen, and V.A. Oboznov, Phys. Rev. B 46, 578 (1992).

${ }^{8}$ O.H. Olsen, A.V. Ustinov, and N.F. Pedersen, Phys. Rev. B 48,
13133 (1993).

${ }^{9}$ M. Cirillo, N. Grønbech-Jensen, M.R. Samuelsen, M. Salerno, and G. Verona Rinati, Phys. Rev. B 58, 14372 (1998).

${ }^{10}$ M. Salerno and M.R. Samuelsen, Phys. Rev. B 59, 14653 (1999).

${ }^{11}$ A. Barone and G. Paternò, Physics and Applications of the Josephson Effect (Wiley, New York, 1982).

${ }^{12}$ R.D. Parmentier, in The New Superconducting Electronics, Vol. 251 of NATO Advanced Studies Institute Series E, Applied Sciences, edited by H. Weinstock and R.W. Ralston (Kluwer, Dordrecht, 1993), p. 221.

${ }^{13}$ N. Grønbech-Jensen and M. Cirillo, Phys. Rev. B 50, 12851 (1994); N. Grønbech-Jensen, P.S. Lomdahl, and M. Cirillo, ibid. 51, 11690 (1995).

${ }^{14}$ E. Jahnke and F. Emde, Tables of Functions (Dover, New York, 1945), p. 145. 